\title{
Trattamento di ampia ferita di gamba con esposizione tendinea con lamine cellulari allologhe: un caso clinico
}

\section{L.Vaienti, M. Brioschi,S.Marcelli,E.Benanti, A. Marchesi \\ Dipartimento di Chirurgia Plastica-Ricostruttiva, I.R.C.C.S Policlinico San Donato, Università degli Studi di Milano}

DOI 10.1007/s10261-013-0063-0

\begin{abstract}
Treatment of a large wound of the leg with tendon exposure by allergenic sheets: a case report

Background Chronic lower limb ulcers still represent a huge cost for health care systems. In addition to traditional surgical and medical techniques, several bioengineered products have been introduced.

We report a case of a post-traumatic wound of the leg, complicated by tendon exposure treated. Due to general conditions of the patient, we chose to avoid major surgical operations and we used an allogeneic keratinocyte sheet on a hyaluronan acid device to improve the wound healing. Results The ulcer successfully healed in 60 days without needing any major operations. Conclusions The mechanisms of action, by which these allogeneic devices are able to improve the wound healing, are still uncertain and have to be studied. Nevertheless, these constructs may represent another option in the field of regenerative surgery for lower extremity wounds.
\end{abstract}

\section{Introduzione}

Nei paesi occidentali è stato calcolato che il 10 per mille della popolazione adulta è stata affetta almeno una volta nella sua vita da un'ulcera agli arti inferiori [1]. Phillips et al. hanno rilevato la perdita di 2 milioni di giornate lavorative a causa di ulcere degli arti inferiori [2]. Molte ulcere possono persistere per anni, nonostante una adeguata terapia topica e sistemica, inoltre possono, una volta guarite, recidivare. Il trattamento delle ulcere degli arti inferiori rappresenta un rilevante problema nel campo della salute sia in termini economici, di invalidità e morbidità, che in termini di complessità ricostruttiva.

Come proposto da Levin nel 1993, le indicazioni chirurgiche per ricostruire un difetto dei tessuti molli deve seguire una "scala" ricostruttiva ben delineata, iniziando dalla chiusura della ferita per prima intenzione fino al lembo microchirugico [3]. Tuttavia, in perdite di sostanzaparticolari sedi, quali l'esposizione tendinea negli arti inferiori, i lembi microchirurgici rappresentano spesso il primo approccio del chirurgo plastico ricostruttore. Aspetti di tale procedura sono la necessaria esperienza nella tecnica microchirurgica, i tempi operatori lunghi e complicanze che possono esitare nella necrosi completa del lembo allestito (circa il 4\%) [4]. Riportiamo un caso di ulcera posttraumatica a livello della gamba, con esposizione tendinea, trattata con impiego di lamina cellulare allologa e innesto dermo-epidermico.

\section{Caso clinico}

Da circa 1 anno la paziente di 92 anni riferisce comparsa di ulcera post-traumatica alla gamba sinistra che non tende alla guarigione e che presentava evento infettivo 10 giorni prima dell'accesso in ambulatorio. In anamnesi patologica remota si segnala ictus cerebri nel luglio 2003 con esito di emiplegia sinistra, diabete mellito lieve, ipertensione arteriosa, colelitiasi. All'esame obiettivo locale si reperta la presenza di vasta lesione al $3^{\circ}$ distale-anteriore della gamba sinistra con esposizione tendinea parzialmente privo di paratenon (Fig. 1).

Si esegue ecocolor-doppler che dimostra grave arteriopatia dell'arto inferiore sinistro con IW 0.45 . Si pone in lista per arteriografia ed eventuale rivascolarizzazione endovascolare. Viene eseguito intervento di toilette della lesione, revisione e tenectomia parziale e copertura con innesto dermoepidermico a spessore parziale. Il decorso postoperatorio segnala il mancato attecchimento dell'innesto dermoepidermico. In seguito a PTA femoro-poplitea rimane una condizione di precaria vascolarizzazione a livello dell'arto inferiore sinistro.

Date le comorbidità, la grave arteriopatia periferica, il rischio anestesiologico che controindicherebbero la copertura dell'ulcera con esposizione tendinea mediante lembi perforanti locali o microchirurgici, si pone indicazione al trattamento della lesione mediante toilette chirurgica e apposizione di lamina di cheratinociti allogenici su scaffold di acido ialuronico esterificato (Fig. 2) A distanza di 7 giorni dall'applicazione della lamina, la lesione appariva ridotta nelle dimensioni, copertura quasi totale dell'esposi-

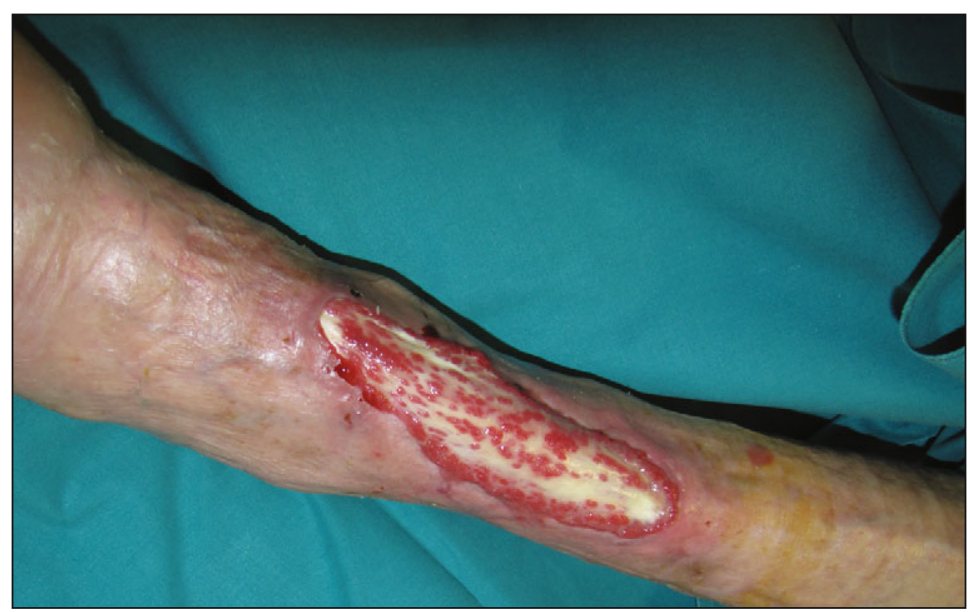

Fig. 1. Immagine preoperatoria con esposizione tendinea priva di paratenon zione tendinea e detersione de
Fig. 2. Immagine intraoperatoria dopo toilette chirurgica

fondo della lesione. Si osserva miglioramento del quadro lesionale a 40 giorni, con superficializzazione marcata del fondo della lesione e margini indenni da flogosi o edema perilesionale. A 60 giorni dal posizionamento della lamina di cheratinociti allogenici la lesione è completamente chiusa e la ferita appare consolidata (Fig. 3).

\section{Discussione}

L'innesto di sostituti epidermici cellulari, ovvero lamine di chera tinociti su scaffold di biomateriale, costituisce oggi un metodo comprovato ed efficace per il trattamento di vaste perdite cutanee.Il flusso produttivo di questi nuovi tessuti ingegnerizzati inizia con il prelievo di cute da pazienti donatori cadavere. La banca della cute - C.R.R. Innesti cutanei (Ospedale Niguarda di Milano) si occupa del reperimento, processazione e conservazione della cute da donatore. Per l'accettazione sono necessari esami sierologici tali da escludere patologie infettive o cutanee (HBsAg anti$\mathrm{HBC} \mathrm{AB}, \mathrm{HBsAb}$, anti HCV Ab,
infettive o cutanee (HBsAg, anti-

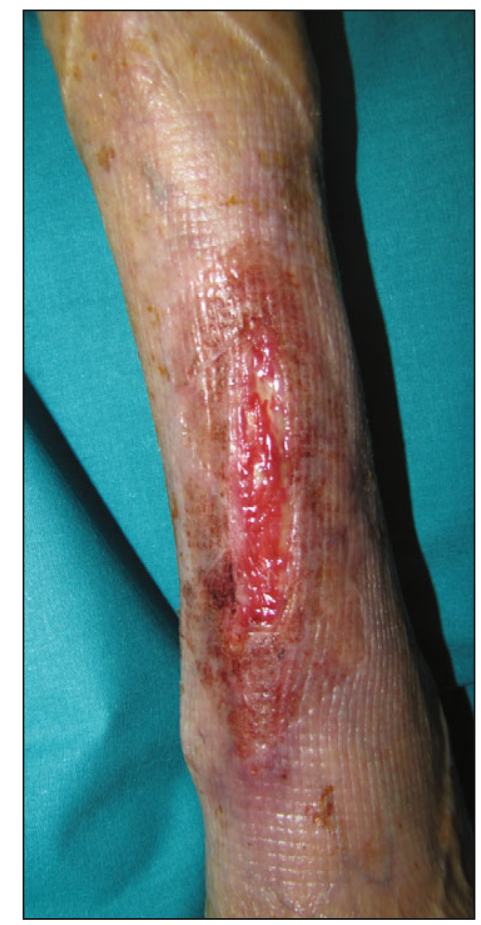

anti-HIV 1 e 2, CMV, TPHA, HTLV-1 test molecolari di screening TRI-NAT: HIV, HBV, HCV) [5]. La biopsia viene sottoposta a processi enzimatici e incubazione per ottenere la quota cellulare. I cheratinocitivengono espansi e coltivati secondo la metodica di Rheinwald\& Green [6]. Raggiunta la confluenza, avviene la pluristratificazione cellulare sul supporto di HYAFF, un estere benzilico dell'acido ialuronico, per ottenere la lamina cellulare [7]. Vengono riportate esperienze se-

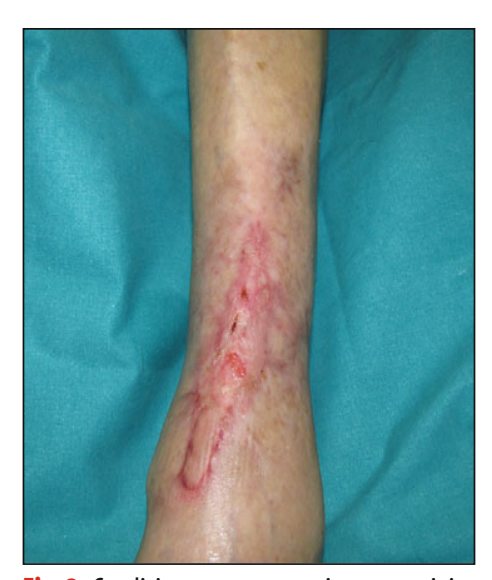

Fig. 3. Condizione postoperatoria con guarigione completa

condo le quali l'epitelio coltivato allogenico non subisca rigetto [8], dovuta alla mancata espressione dell'antigene di classe II nei cheratinociti prodotti in vitro. Sul meccanismo d'azione si è supposto che l'alloinnesto potesse attecchire cosi come avviene per gli innesti dermoepidermici, in modo permanente [9]. Tuttavia studi che hanno utilizzato analisi di DNAe prove cromosomiche, hanno dimostrato che dopo circa una settimana non si osserva alcun cheratinocita donatore [10] nel sito di applicazione delle lamine, indicando che l'alloinnesto funzioni stimolando la migrazione e la proliferazione dei cheratinociti dell'ospite stesso. La lamina allogenica agirebbe producendo fattori di crescita per le cellule epiteliali e dermiche dell'ospite, inducendo la guarigione tissutale [11]. Lacido ialuronico viene liberato dal contatto dell'estere con l'essudato della ferita. Dato che la guarigione fetale, che avviene mediante rigenerazione dalle cellule proprie del tessuto leso, è caratterizzata da alte deposizioni di acido ialuronico, è possibile che la lamina allogenica promuova una guarigione tissutale simile a quella fetale, con resistutio ad integrum [12] della lesione.

La scala ricostruttiva, nella sua versione classica, rimane una guida indispensabile per il chirurgo plastico ricostruttore. L'esposizione tendinea rappresenta tuttora una sfida ricostruttiva nel terzo medio-distale di gam-

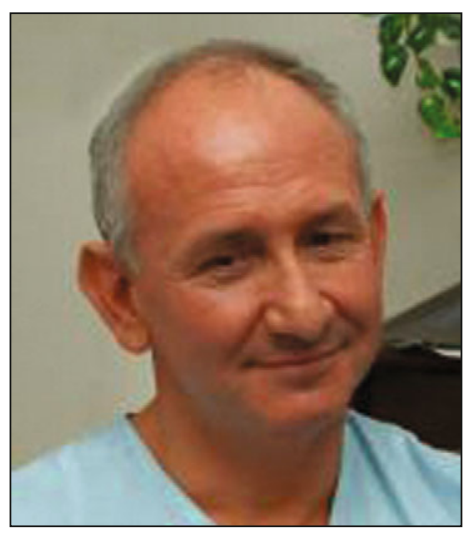

L. Vaienti

ba, e le nuove lamine su scaffold di acido ialuronico si sono rivelate una utile alternativa ad unità ricostruttive più invasive, portando in tempi rapidi alla risoluzione del quadro clinico.

\section{Bibliografia}

Wester W (1993) Legulcers: diagnosis and treatment. Elsevier

Philips et al. (1994) A study of the impact of legulcers on quality of life: financial, social and psychologicimplications. J AmAcadDermatol 31: 49-53 Levin LS. (1993) The reconstructiveladder: an orthoplasticapproach. OrthoClin North Am 24:393e409

Khouri RK, Cooley BC, Kunselman AR, et al (1998) InternationalMicrovascularRe-

searchGroup. Aprospectivestudyofmicrovascular free-flap surgery and outcome. PlastReconstrSurg 102:71le21

Linee Guida per il prelievo, la conservazione e l'utilizzo di cute e dei prodotti dell'ingegneria dei tessuti a scopo di trapianto. All, 1 Suppl. Straordinario al n 17, Boll Uff Regione Lombardia - 24 Aprile 2003

Rheinwald JG Green H. (1975) Serial cultivation of strains of human epidermalkeratinocytes: The formation of keratinizingcolonies from single cells. Cell 6:331-343

Andreassi L., Casini L, Donati L (1991) Human KeratinocytesCultured on MembranesComposed of Benzyl Ester of Hyaluronic Acid Suitable for GraftingWounds. 3:116-126

Phillips TJ, Bhawan J, Leigh IM et al (1990) Culturedepidermalautografts and allografts: a study of differentiation and allograftsurvival. J AmAcadDermatol 23:189-98

Morhenn VB, Benike CJ, Cox AJ, et al (1982) Cultured human epidermalcells do notsynthesize HLA-DR. J InvestDermatol 78: 32-7

10. Brain A, Purkis P, Coates P, et al (1989) Survival of culturedallogeneickeratinocytestran-

splanted to deepdermal bed as- 